\title{
Mycotic Aneurysm: A Diagnostic and Therapeutic Challenge
}

\author{
João Cruz*, Lameiras R, Figueiredo F, Costa J, Lourenço $R$ and Ramalho $M$
}

Radiology Department, Hospital Garcia de Orta, Almada, Portugal

*Corresponding author: João Cruz, Radiology Department, Hospital Garcia de Orta, Av. Torrado da Silva, 2801-951, Almada, Portugal, Tel: +3519-3217-3616,_E-mail:.jpr_cruz@hotmail.com

\begin{abstract}
Mycotic or infected aneurysms (MA) are rare, accounting for only $1-3 \%$ of all arterial aneurysms, and usually affect major arteries. Visceral MA are the least frequent and most commonly involve the superior mesenteric artery. MA are associated with a high morbidity and mortality, estimated in 19\% within 6 months of diagnosis despite surgical or endovascular intervention. Early treatment is essential to reduce mortality, but initial symptoms are often insidious and non-specific, hampering the diagnosis. Imaging plays a key role in MA diagnosis with contrast-enhanced CT allowing confident identification and characterization of vascular anatomy and anomalies.

The authors present the case of a 75-year-old woman who presented to the emergency department (ED) with left flank pain irradiating to the epigastrium for 2 weeks, with signs and blood tests suggestive of infection, and with imaging evidence of splenic abscesses and a celiac trunk aneurysm. During the hospital stay, a conservative approach was attempted, but the aggravation of clinical and laboratorial parameters, combined with imaging evidence of expanding mycotic aneurism led to endovascular correction of the celiac trunk mycotic aneurysm. The patient had post-operative aggravation of clinical parameters and died 3 days later from cardiorespiratory arrest.
\end{abstract}

\section{Keywords}

Infected aneurysm, Celiac artery, Spiral computed tomography

\section{Case Report}

We report the case of a 75 -year-old woman who presented to the ED with left flank pain irradiating to the epigastrium for 2 weeks, without significant relief despite taking strong painkillers (Tramadol + Paracetamol).

She had personal history of atrial fibrillation, high blood pressure, grade $V$ chronic kidney disease on hemodialysis, carotid artery stenosis, bilateral peripheral arterial disease and previous stroke without major neurological deficits. She was medicated with Clopidogrel, Carvedilol, Rosuvastatin, Lorazepam and Esomeprazole.

In the E.D., the patient was drowsy but oriented, febrile, with axillary temperature of $39^{\circ} \mathrm{C}$. On physical examination, the abdomen was soft, depressible and painless on palpation.

Blood tests showed elevated white blood cell count, $27.8 \times 10^{9} /$ L (reference value [RV]: $4-11 \times 10^{9} / L$ ), with 90.6\% neutrophils (RV: 40-74\%), and elevated reactive C-protein (RCP), $14.2 \mathrm{mg} / \mathrm{dL}$ (RV: $<0.2 \mathrm{mg} / \mathrm{dL}$ ).

An abdominal ultrasound was performed, depicting a splenic nodule with predominantly cystic echotexture. It was also seen an image suggestive of a celiac trunk aneurysm, with hyperechogenicity of adjacent fatty tissues.

A computed tomography (CT) was then requested (Figure 1), confirming the presence of two splenic hypovascular nodules, suggestive of collections, as well as the presence of a saccular dilatation of the proximal segment of the celiac trunk, with $13 \times 12 \times 10 \mathrm{~mm}$ (transversal $\times$ antero-posterior $\times$ longitudinal diameters, respectively).

The patient was admitted with the hypothesis of splenic abscesses vs. infected splenic infarctions, to undergo conservative management, starting antibiotherapy with Piperacillin, Tazobactam and Metronidazol.

During the hospital stay, the patient presented more prostrated and disoriented, with labile values of blood pressure, without relief of abdominal pain. Meanwhile, blood cultures were positive for Streptococcus agalactiae with sensitivity to penicillin.

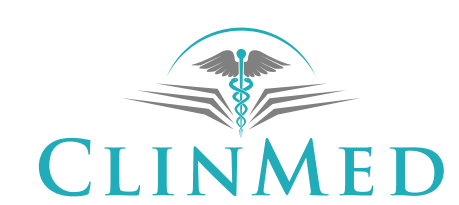

INTERNATIONAL LIBRARY
Citation: Cruz J, Lameiras R, Figueiredo F, Costa J, Lourenço R, et al. (2018) Mycotic Aneurysm: A Diagnostic and Therapeutic Challenge. Int J Radiol Imaging Technol 4:036. doi.org/10.23937/2572-3235.1510036 Accepted: June 04, 2018: Published: June 06, 2018

Copyright: (c) 2018 Cruz J, et al. This is an open-access article distributed under the terms of the Creative Commons Attribution License, which permits unrestricted use, distribution, and reproduction in any medium, provided the original author and source are credited. 


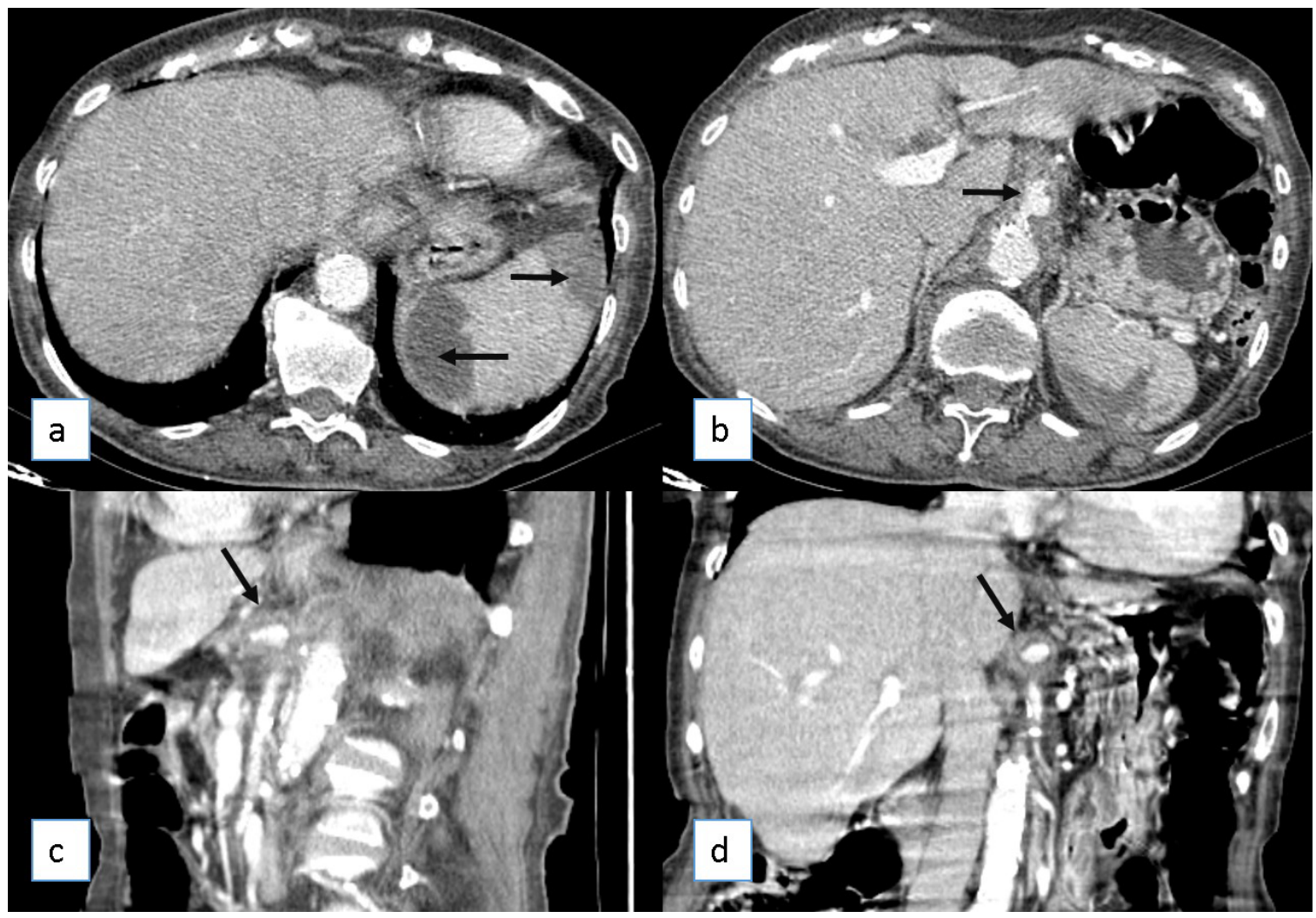

Figure 1: Axial (a,b), sagittal (c) and coronal (d) reformations of CT after injection of iodine contrast agent. Several hypovascular areas are depicted (arrows in a) in splenic peripheral topography; b), c) and d) a saccular dilatation of the proximal segment of the celiac trunk is depicted (arrows) in the three planes.

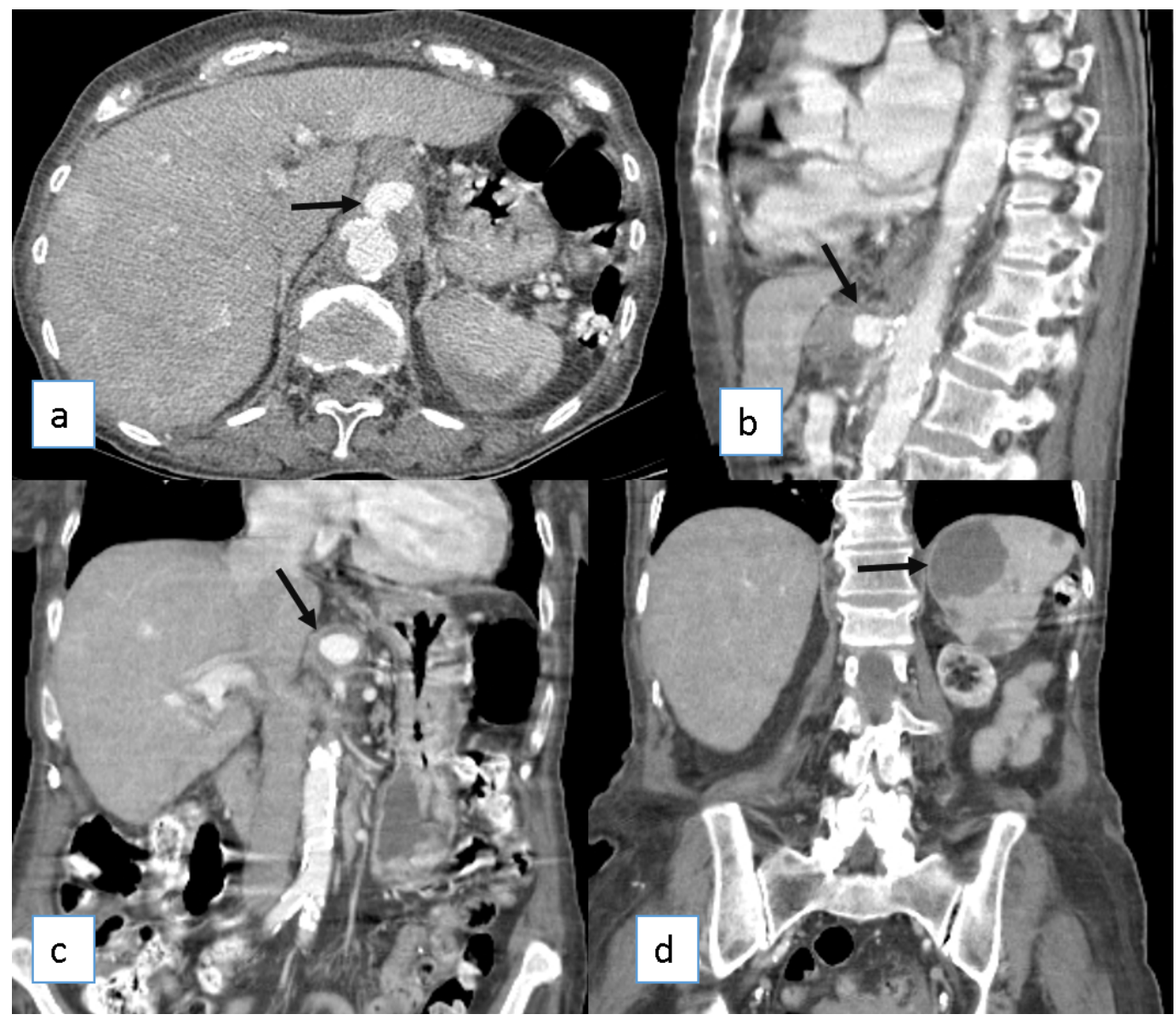

Figure 2: Axial (a), sagittal (b) and coronal (c,d) reformations of CT after injection of iodine contrast agent. This examination, performed 3 days after the former, shows clear expansion of the celiac artery aneurysm (arrows in a,b,c). The hypovascular splenic areas (arrow in d) were remained unchanged. 
As there was an aggravation of clinical and laboratorial parameters, a re-evaluation CT was performed 3 days later (Figure 2), revealing expansion of the celiac trunk aneurysm $(21 \times 17 \times 16 \mathrm{~mm})$, with accentuated stranding of adjacent fatty tissues, suggesting an infectious/mycotic etiology. Nevertheless, hepatic and gastroduodenal arteries showed normal permeability. Several reactive enlarged mesenteric lymph nodes were also present. The splenic abscesses showed no significant aggravation.

The splenic abscesses were assumed to be secondary to the MA and the patient was proposed to undergo endovascular correction of the celiac trunk mycotic aneurysm, which was performed 15 days after the admission, with exclusion of the aneurysm with a bifurcated endoprosthesis to the superior mesenteric artery.

The patient had a difficult post-operative course, with aggravation of clinical and laboratorial parameters, with refractory hypotension and anemia, despite repeated transfusions and aminergic medication.

A re-evaluation CT was performed 3 days after the endovascular surgery (Figure 3 ), where the bifurcated endoprosthesis is depicted, without visible blood circulation on celiac trunk nor its branches. There were also signs of de novo ischemic liver infarcts and new splenic lesions.

The patient died 3 days later from cardiorespiratory arrest.

\section{Acknowledgement}

Mycotic or infected aneurysms (MA) are rare in today's clinical practice due to effective and prompt antibiotic therapy, accounting for only 1-3\% of all arterial aneurysms, but they can affect any artery and be potentially life threatening [1,2]. MA usually affect major arteries, classically at branch points and the aorta, peripheral arteries, cerebral arteries, and visceral arteries are involved in descending order of frequency [1]. Visceral
MA are rare and most commonly involve the superior mesenteric artery, representing only $10 \%$ of all visceral aneurysms, most commonly caused by atherosclerosis [2-4]. MA is associated with a high morbidity and mortality, estimated in $19 \%$ within 6 months of diagnosis despite surgical or endovascular intervention [5].

The pathogenesis of MA involves arterial wall degeneration, hastened by infiltration of immune cells secondary to bacterial infection which may arise through hematogenic seeding from distant septic foci, lymphatic spread, contiguous extension or direct inoculation (iatrogenic during angiography or through intravenous drug misuse) [2,5].

Classic clinical presentation involves fever, pain referable to the aneurysm site, and elevated white blood cell count (WBC). Other initial symptoms include a febrile illness with insidious onset, general malaise and weight loss. Those presenting late may manifest with profound septicemia or with consequences of rapid aneurysm expansion or rupture $[2,5]$.

Early diagnosis and treatment is essential to reduce mortality, but diagnosis is often elusive due to the non-specific initial symptoms. Therefore, a high level of suspicion is needed to prompt accurate diagnosis [5].

Over time, imaging evaluation shifted from transcatheter angiography to cross sectional imaging, primarily with computed tomography angiography (CTA) and occasionally magnetic resonance angiography (MRA). Allowing a fast exploration of the systemic arterial vasculature with detailed multiplanar and three-dimensional angiographic reconstructions, the volumetric contrast-enhanced CT allows confident identification and characterization of vascular anatomy and anomalies [3]. Deipolyi, et al. [5] in their series refer that imaging was considered diagnostic in $75 \%$ of cases.

Because rapid progression of arterial wall degeneration related to its inflammatory etiology occurs, MA typically appears as a saccular outpouching that changes

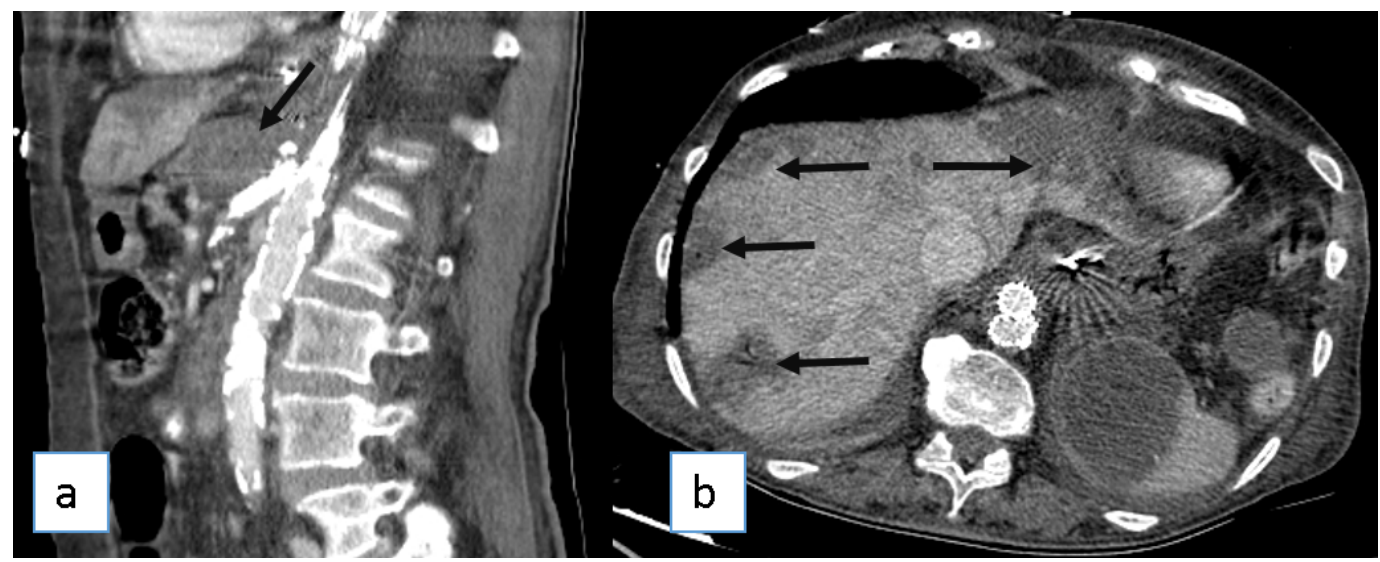

Figure 3: Sagittal (a) and axial (b) images of CT after injection of iodine contrast agent. In this re-evaluation CT performed 3 days after the endovascular surgery, a bifurcated endoprothesis is present in aorta and superior mesenteric artery, without visible blood circulation on celiac artery (arrow in a) nor its branches. There were also signs of de novo ischemic liver infarcts (arrows in b). 
over time. Other imaging features suggestive of infected aneurysm can include indistinct or irregular arterial wall and perivascular soft tissue changes, inflammation, gas and edema [3,5]. A characteristic feature of mycotic aneurysms is their rapid development and unpredictable behavior: These lesions may undergo partial or complete thrombosis as a response to medical treatment, decrease in size or otherwise enlarge [3].

Treatment of MA involves intravenous antibiotics and most frequently surgical intervention with bypass grafts. Endovascular repair, while controversial, has been used with success. Despite available treatments, mortality remains high, particularly in the case of aneurysmal rupture [5].

In our case, the presence of several comorbidities and the delay in searching for medical assistance may justify the poor outcome of our patient, highlighting the poor prognosis of MA when not recognized and/or treated within the proper timing. For the interpreting radiologist, a focal saccular arterial dilatation with perivascular inflammation should trigger a thorough search of the medical re- cord and discussion with the referring provider to evaluate the possibility of MA, particularly if the aneurysm is noted to change rapidly over serial studies [5].

\section{Conflicts of Interest}

The authors report no conflict of interest.

\section{References}

1. Lee W, Mossop PJ, Little FA, Fitt GJ, Vrazas JI, et al. (2008) Infected (mycotic) an-eurysms: Spectrum of imaging appearances and management. Radiographics 28: 1853-1868.

2. Fisk M, Peck LF, Miyagi K, Steward MJ, Lee SF, et al. (2012) Mycotic aneurysms : A case report, clinical review and novel imaging strategy. QJM 105: 181-188.

3. Tonolini M, Petullà M, Bianco R (2012) Mycotic visceral aneurysm complicating infectious endocarditis: Imaging diagnosis and follow-up. J Emerg Trauma Shock 5: 201-203.

4. Kordzadeh A, Kalyan JP, Jonas A, Hanif MA, Prionidis I (2015) Cryptogenic mycotic aneurysm of the superior mesenteric artery. J Surg Case Rep 2015: 106.

5. Deipolyi A, Bailin A, Khademhosseini A, Oklu R NU SC (2015) J Clin Imaging. 